\title{
OVERTURNING PROBABILITY OF RAILWAY VEHICLES UNDER WIND GUST LOADS
}

\author{
Carsten Proppe and Christian Wetzel \\ Institut für Technische Mechanik, Universität Karlsruhe, Kaiserstr. 12, 76128 Karlsruhe, \\ Germany, Phone: +49 721608 6822, Fax: +49 721608 6070, Email: proppe@itm.uka.de
}

\begin{abstract}
Sufficient crosswind stability is an important criterion in the approval process of railway vehicles. However, crosswind stability is in conflict with demands for light-weight constructions (especially cabin cars) and higher driving velocities. In many countries, the approval process foresees stability predictions based on worst case scenarios, where uncertainties are taken into account by means of safety factors and comparison with reference vehicles. This procedure is a burden for innovations and hinders the interoperability of railway vehicles. Therefore, models have been proposed that take some of the uncertainties associated with the wind gusts and the aerodynamic coefficients of the carbody into account. In this paper, a consistent stochastic wind gust model is proposed, and probabilistic characteristic wind curves are computed by means of a reliability analysis of the train-environment system.
\end{abstract}

Key words: crosswind stability, wind gusts, reliability analysis

\section{INTRODUCTION}

Recent developments in railway engineering have been showing a trend to faster, more energy efficient and more comfortable trains with a higher capacity of passenger transportation. These efforts are directly leading to light-weight cars with distributed traction. Unfortunately, these developments significantly alter the crosswind stability in a negative manner.

Therefore, crosswind stability has become a crucial issue of modern railway vehicle design that cannot be solved easily as all counter-measures 
are very expensive. If a railway vehicle fails to be certified, ballasting is often the only possible solution.

During the last 140 years about thirty wind-induced accidents have been reported. Most of these accidents happened in Japan and Switzerland on narrow gauges at highly endangered points (e.g. bridges or embankments) in nearly hurricane conditions [8-9]. But also on standard gauge track, incidents were reported.

Due to the desired interoperability in Europe the leading operating companies of trains are working on Technical Specifications for Interoperability (TSI) to get a common rule for the certification of railway vehicles [19]. At the moment, most of the leading operating companies of trains in Europe are using approval processes which are based on worst case scenarios where uncertainties are taken into account by means of safety factors [14-15]. They are based on characteristic wind curves obtained from deterministic wind scenarios. The first formulation of this approach is generally attributed to Cooper [6]. Uncertainties enter only during the subsequent risk assessment process, where the risk of an incident is quantified based on a specific vehicle and connection. [1]. Taking uncertainties underlying the computation of the characteristic wind curve into account, Carrarini[4], for the first time, proposes a probabilistic characteristic wind curve.

In this paper the vehicle-environment system is analysed and a model for the crosswind analysis is proposed that includes the most significant uncertainties. They are represented as random variables, whose distributions are determined based on available data in the literature. Finally, crosswind stability is expressed as probability of failure, which can be computed by means of analytical or numerical approaches.

The paper is organized as follows: the following section discusses the adopted model for the vehicle-environment system in detail. After that, the simulation procedure is introduced. In section 4 , a representative cabin car is studied and the principle results are briefly stated, while section 5 contains the major conclusions.

\section{MODELING OF THE SYSTEM}

The system under study consists of two parts:

- the multibody vehicle model; 
- the environmental model.

The environmental model itself has two distinct components: the track (interaction with the vehicle model by means of the wheel/rail contact) and the aerodynamic model (interaction with the vehicle model by means of aerodynamic forces). The subsequent sections are dedicated to an in depth discussion of crucial modeling assumptions.

\section{$2.1 \quad$ Vehicle model}

Commercial multi-body system software has been employed in order to accurately represent the vehicle, a cabin car. The elasticity of the carbody and the bogie frames has been neglected. On the other hand, nonlinearities of the spring and damper characteristics and the bump stops have been carefully taken into account. The latter are responsible for the orientation of the bogies during application of the wind loads. The train is assumed to move with constant velocity on the track.

\section{$2.2 \quad$ Environmental model}

\subsubsection{Track model}

Sections of straight and curved track with constant cant deficiency have been investigated. The track is fitted with UIC 60 rails at standard track gauge of $1435 \mathrm{~mm}$. Excitation by means of measured track irregularities of an intermediate quality German railway has been considered, in order to take effects of long wave track irregularities into account. The sleepers were modeled as rigid bodies. An elastic contact model has been adopted; the tangential forces have been computed by means of Kalkers Fastsim algorithm [12].

Vehicle overturn is described as critical wheel unloading, i.e. by the condition

$$
\frac{Q}{Q_{0}} \leq \delta Q,
$$

where $Q_{0}$ is the static wheel load, $Q(t)$ the actual wheel load and $\delta Q$ a safety margin usually taken as $10 \%$ or $5 \%$. 
Other criteria, such as flange climbing (ratio of lateral to vertical wheel force) and track shift (sum of lateral forces on each axle) are less critical [14], when large aerodynamic loads are acting on the train.

\subsubsection{Aerodynamic model}

The model for the crosswind consists of a superposition of the mean wind, the gust characteristic and the turbulent fluctuations. As the train speed is much higher than the velocity of the crosswind, the spatial correlation of the wind is neglected. Thus, the wind excitation is modeled, as if the train were running through a frozen wind field. Hence, the actual wind speed is a function of the track variable $s$. This function is transformed into the time domain by means of a reference velocity, which is the train speed $v_{0}$.

Two wind scenarios are investigated:

- a train coming out of a tunnel immediately being hit by a gust;

- a train traveling on an embankment under constant mean wind load being hit by a gust.

Various shapes of the wind gust have been proposed in the literature. They have been reviewed and critically discussed in [5]. While former standards preferred '1-cos' gust shapes [10-11], there are strong theoretical arguments [2] in favor of an exponential shape (cf. Fig. 1) of the gust. However, as Carrarini [5] pointed out, direct computation of the aerodynamic forces and moments under assumption of stationary aerodynamics would lead to artificial results (and moreover pose severe problems to a correct numerical integration of the equations of motion). Taking unsteady aerodynamics via the aerodynamic admittance into account amounts to the application of a low pass filter (or a moving average) to the gust velocity time series. The gust shape introduces two parameters: gust amplitude and gust duration. They are represented as positive random as discussed in [7].

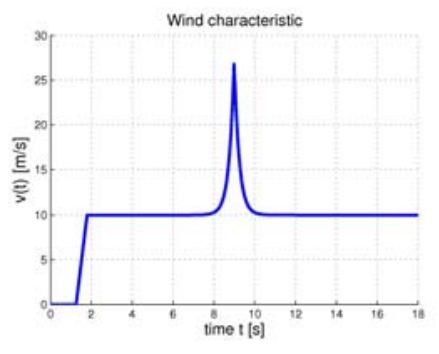

Figure 1. Representative mean gust shape. 
Turbulent fluctuations of the wind velocity are computed by a spectral decomposition of the von-Kármán spectral density function (cf. Fig. 2), which is often used to describe air turbulence [20]. The turbulent fluctuations are assumed to be normally distributed.

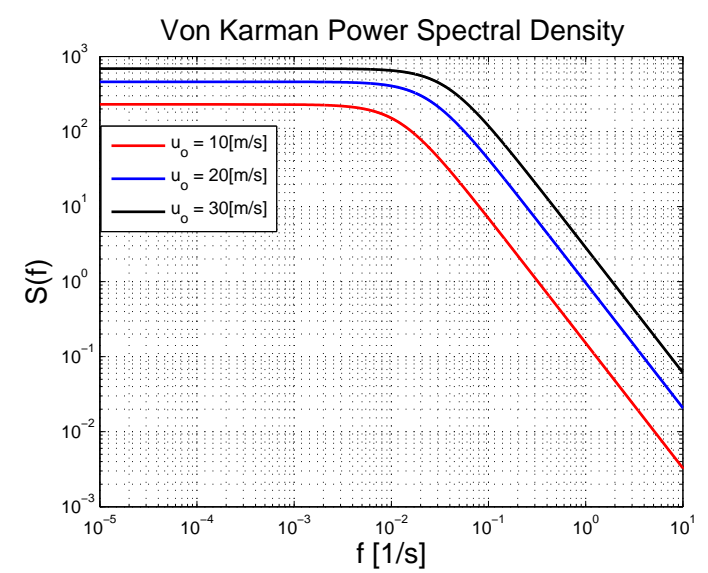

Figure 2. von Kármán spectral density

The resultant wind velocity $v(t)$ is thus the sum of three different parts: mean wind, gust characteristic, and turbulent fluctuations. Similar approaches are common in the design of wind turbines [2].

The wind loads exerted on the vehicle are represented as concentrated loads. The coordinate system and the composition of the wind velocity vector acting on the train are shown in Fig. 3.

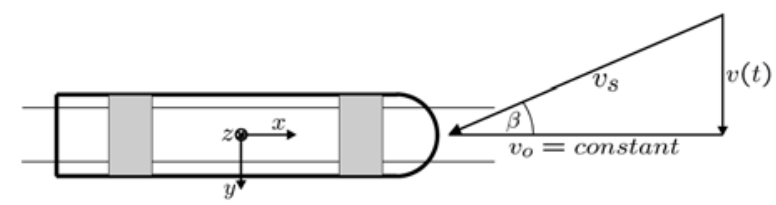

Figure 3. Coordinate system and wind velocity vector

The aerodynamic forces and moments are computed from the acting wind velocity $v_{s}(t)$ by means of experimentally determined aerodynamic coefficients: 


$$
\begin{aligned}
& F_{y / z}(t)=\frac{1}{2} C_{\text {side } / \text { lift }}(\beta) \rho A v_{s}^{2}(t), \\
& M_{x / y / z}(t)=\frac{1}{2} C_{\text {roll / pitch } / \text { yaw }}(\beta) \rho A l v_{s}^{2}(t) .
\end{aligned}
$$

The factors $A$ and $l$ are the related area and length dimension of the vehicle, resp. As the influence of the drag force on the crosswind stability is negligible the drag coefficient is not considered. The aerodynamic coefficients $C_{\text {side/liftroll/pitch/yaw }}$ depend nonlinearly on the angle

$$
\beta=\tan ^{-1}\left(\frac{v(t)}{v_{0}}\right)
$$

For the calculation of $v_{s}(t)$, oscillations of the carbody are neglected and only the reference velocity is taken into account, as they are much smaller than the wind velocity. The aerodynamic coefficients are assumed to be random variables. Very little is known about their distribution [5], and their correlation is completely unknown. From the available experimental data, a normal distribution with a coefficient of variation of $10 \%$ seems to be reasonable.

\section{SIMULATION CONCEPT}

For the calculation of the probability of failure $P_{f}$, it is necessary to evaluate integral

$$
P_{f}=\int_{\Omega_{f}} p_{Z^{*}}\left(z^{*}\right) d z^{*}
$$

over the failure domain $\Omega_{f}$, where $z^{*}$ is the array of all stochastic variables of the system and $p_{Z^{*}}\left(Z^{*}\right)$ the joint probability density function. The failure domain $\Omega_{f}$ is the set of all arrangements of $z^{*}$ which forces the wheelunloading $\delta Q$ to fall below the safety margin. For such a complex multibody system, the failure domain is not known explicitly but can only be evaluated pointwise. The integral in (3) can be simplified by using the law of conditional probability. The probability of failure is then obtained as 


$$
P_{f}=\int_{u_{0, d}}^{u_{0, t}} P\left(z \mid u_{0}\right) p\left(u_{0}\right) d u_{0},
$$

where $P\left(z \mid u_{0}\right)$ is the failure probability conditioned to the mean wind speed $u_{0}, p\left(u_{0}\right)$ the pdf of $u_{0}$ and $z$ the array $z^{*}$ without $u_{0}$. In order to evaluate (4), $P\left(z \mid u_{0}\right)$ and $p\left(u_{0}\right)$ have to be known. The latter can be obtained from meteorological measurements, while the former has to be computed.

For simplification, the calculation of $P\left(z \mid u_{0}\right)$ is only done at certain predefined mean wind velocities which reduces the evaluation of (4) to the computation of the finite sum

$$
P_{f}=\sum_{i=1}^{N} P\left(z \mid u_{0, i}\right) p\left(u_{0, i}\right) \Delta u_{0, i},
$$

The conditional failure probability $P\left(z \mid u_{0}\right)$ can be evaluated either by analytical methods, such as FORM or SORM [18] or by numerical methods employing Monte Carlo simulation with variance reduction [17], eventually under application of a response surface [3]. Here, all distributions are mapped to a standard Gaussian space, in which the shortest distance to the failure domain, the so called design point, is computed. After that, importance sampling around the design point and line sampling [16] has been employed in order to obtain reliable estimates of the conditional failure probability.

\section{4. $\quad$ RESULTS}

Preliminary studies indicated that the influence of the turbulence on the probability of failure can be neglected. Therefore, the following results refer to a wind model without atmospheric turbulence.

Figure 4 shows the conditional failure probability versus the mean wind speed for the tunnel exit wind scenario for a typical cabin car traveling with $160 \mathrm{~km} / \mathrm{h}$ on straight track. It can be clearly seen that the differences between the results obtained by FORM and the sampling based results are large (about 30\%). The results show an exponential increase of the failure probability with increasing wind speed over a range of several orders of magnitude. 


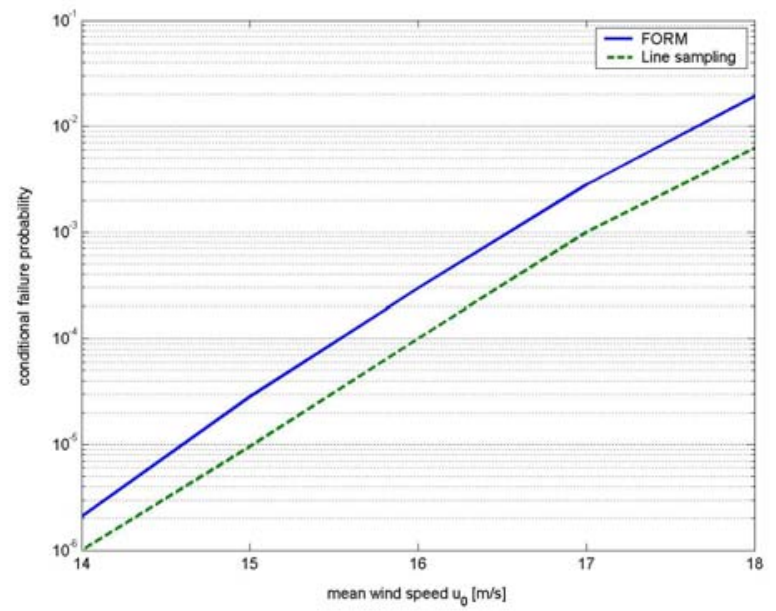

Figure 4. Conditional failure probability vs. mean wind speed for tunnel exit scenario.

Figure 5 compares the conditional failure probability for the tunnel exit and the embankment scenario. As can be expected, failure probabilities for the embankment scenario are lower than for the tunnel exit. Stated in an other way, the cabin car can sustain mean wind speeds that are approximately $2 \mathrm{~m} / \mathrm{s}$ higher at the same failure level.

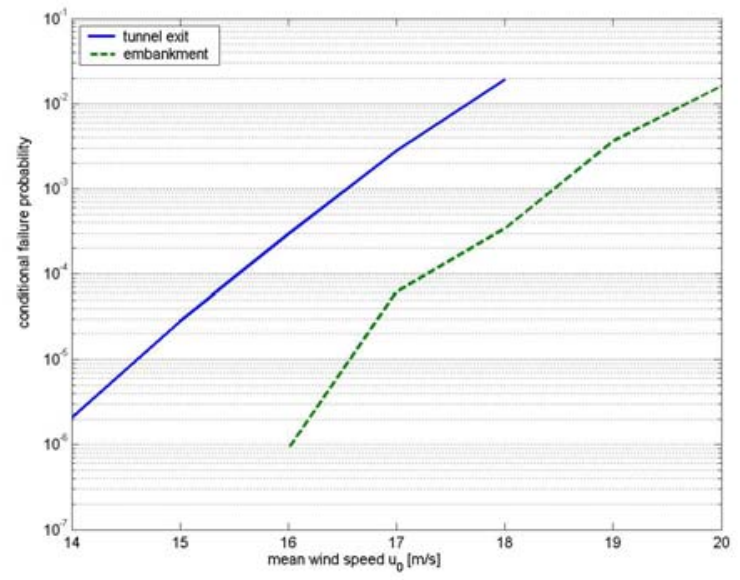

Figure 5. Comparison of failure probability for tunnel exit and embankment scenario (FORM results). 


\section{CONCLUSIONS}

In recent years due to the modern light weight constructions and due to the increasing interoperability in Europe, the crosswind stability of highspeed trains has come to the fore of the leading operating companies of rolling stock. To prove the crosswind stability of a railway vehicle, the state of the art is to calculate the deterministic characteristic wind curve and then to compare this characteristic wind curve with a reference model of an already existing vehicle.

In contrast to this standard procedure, a consistent stochastic approach is proposed in which a probabilistic characteristic wind curve has to be computed. Two wind scenarios, referring to a tunnel exit and a situation on an embankment, are defined. In this way, the most important uncertainties of the vehicle-environment system are accounted for. By prescribing an acceptance level for the probability of failure conditioned to the mean wind speed, a critical mean wind speed can be inferred.

For the first time, a probabilistic characteristic wind curve based on sampling techniques has been computed. Due to the use of very efficient variance reducing sampling algorithms, the effort is not much higher than for the computation of the design point. The results indicate that deviations from the FORM results are large. It is noted that for the cases under investigation, the failure probability increases exponentially with increasing mean wind speed.

Further efforts are necessary in order to clarify the uncertainty modeling of the aerodynamic coefficients and the influence of non-stationary aerodynamics. For the former, due to the lack of data, resort to nonparametric models might by an interesting alternative. Furthermore, it is noted, that the applied procedures are still to complicate in order to enter design codes. However, sensitivity analyses of the conditional probability of failure may lead to considerable simplifications.

Finally, the numerical techniques applied in this study can be generalized in order to couple reliability analysis and multi body system. In this way, a general framework for the uncertainty analysis of multi body systems can be obtained. 


\section{REFERENCES}

1. Andersson E, Häggström J, Sima M, Stichel S. "Assessment of train-overturning risk due to strong cross-winds", J. of Rail and Rapid Transit, 218, pp. 213-223, 2004.

2. Bierbooms W, Cheng PW. "Stochastic gust model for design calculations of wind turbines", Wind Engineering and Industrial Aerodynamics, 90, 1237-1251, 2002.

3. Bucher CG, Burgound U. "A fast and efficient response surface approach for structural reliability problems", Structural Safety, 7, pp. 57-66, 1990.

4. Carrarini A. "A probabilistic approach to the effects of cross-winds on rolling stock", Proc. European Congress on Computational Methods in Applied Sciences and Engineering, Jyväskylä, Finland, 24-28 July, 2004.

5. Carrarini A. Reliability based analysis of the crosswind stability of railway vehicle, Dissertation Thesis, TU Berlin, 2006.

6. Cooper RK. "The probability of trains overturning in high winds", Proc. $5^{\text {th }}$ Int. Conf. on Wind Engineering, Fort Collins, Colorado, USA, July, 1979, pp. 1185-1194.

7. Delaunay D, Locatelly JP. "A gust model for the design of large horizontal axis wind turbines: completion and validation", Proc. European Community Wind Energy Conference, Madrid, Spain, Sep. 10-14, 1990, pp. 176-180.

8. Fujii T, Maeda T, Ishida H, Imai T, Tanemoto K, Suzuki, M. "Wind-Induced Accidents of Train/Vehicles and Their Measures in Japan", Quarterly Report of Railway Technical Research Institute, 40, 1999.

9. Gawthorpe RG. "Wind effects on ground transportation." J. Wind Engineering Industrial Aerodynamics, 52, p. 73-92, 1994.

10. Hoblit FM. Gust Loads on Aircraft. AIAA, Washington DC, USA, 1988.

11. IEC 61400-1 Wind Turbine Safety and Design, 1993.

12. Kalker JJ. "A fast algorithm for the simplified theory of rolling contact", Vehicle System Dynamics, 102, pp. 1-13, 1982.

13. Lippert S. On side wind stability of trains. Report, Royal Institute of Technology Railway Technology, Stockholm, 1999.

14. Lippert S, Tengstrand H, Andersson E., Stichel S. "The effect of strong cross winds on rail vehicles", VDI Berichte, 1568, 2000, pp. 221-241.

15. Matschke G, Grab M, Bergander B. "Nachweis der Sicherheit im Schienenverkehr bei extremem Seitenwind", Betrieb und Verkehr, 51, pp. 200-206, 2002.

16. Pradlwarter HJ, Pellissetti MF, Schenk CA, Schuëller GI, Kreis A, Fransen S, Calvi A, Klein M. "Realistic and efficient reliability estimation for aerospace structures", Computer Methods in Applied Mechanics and Engineering, 194, pp. 1597-1617, 2005.

17. Proppe C, Pradlwarter HJ, Schuëller GI. "Equivalent linearization and Monte Carlo simulation in stochastic dynamics", Probabilistic Engineering Mechanics, 18, pp. 1-15, 2003.

18. Rackwitz R. "Reliability analysis - a review and some perspectives", Structural Safety, 23, p. 365-395, 2001.

19. Schulte-Werning B, Gregoire R, Malfatti A. TRANSAERO - A European Initiative on Transient Aerodynamics for Railway System Optimization, Springer, Berlin, 2002

20. Simiu E, Scanlan RH. Wind Effects on Structures, Wiley, 1996. 